\title{
Hybrid Processes for Extraction-separation of Tocopherols from Crude Palm Oil
}

\author{
K. Vachiraanan ${ }^{1}$, J. Rattanapisit ${ }^{2^{*}}$ \& P. Sridang ${ }^{3}$ \\ ${ }^{1 \& 2}$ Department of Chemical Engineering, Prince of Songkla University (PSU), Songkhla 90112,Thailand \\ ${ }^{3}$ Department of Civil Engineering, Membrane Science and Technology Research Center, Prince of Songkla \\ University(PSU), Songkhla 90112, Thailand
}

\begin{abstract}
TThe aim of this study was to evaluate the hybrid processes for extraction and separation of tocopherols from crude palm oil. The saponification method was used to extract crude palm oil under various concentrations of alcoholic $\mathrm{KOH}$ in the range of $10-60 \% \mathrm{w} / \mathrm{v}$ in a lab scale unit. The experiments were conducted at a temperature and saponification time in the range of $60-80^{\circ} \mathrm{C}$ and $20-40 \mathrm{~min}$ respectively. The performance and efficiency of ultrafiltration were investigated by filtering a fraction of unsponifiables. The results showed that the optimum condition of saponification was $10 \%$ alcoholic $\mathrm{KOH}$ with the minimum loss of $\alpha$-tocopherol of around $19 \%$. A lab scale filtration unit with a hydrophobic sheet membrane was operated under a frontal filtration mode at room temperature. The results pointed out that ultrafiltration could remove most of the residual triglycerides and phospholipids contained in unsponifiables at a TMP of $0.25-1.0$ bars. The removal of up to $70 \%$ of triglycerides and phospholipids was reached when the mixture was ultrafiltered. In addition, these hydride processes present a one of the great option for $\alpha$-tocopherol purification losing about $5 \%$ of yield.
\end{abstract}

Keywords: Crude palm oil, tocopherol, saponification, ultrafiltration

\subsection{INTRODUCTION}

Crude vegetable oils are constituted mainly of triglycerides but contain some minor components such as phospholipids, FFAs, coloring pigments and phytonutrients as carotenes, vitamin $\mathrm{E}$ (tocol, tocopherols and tocotrienols), and sterols [1]. It was reported that crude palm oil consists of $1 \%$ phytonutrients with a tocopherol content of 300$1000 \mathrm{ppm}$. In comparing these phytonutrient groups it has been found that vitamin $\mathrm{E}$ is value added and is the product for which there are increasing demands all over the world. Vitamin $\mathrm{E}$ is a natural antioxidant that plays significant roles in food preservation and disease prevention [2-4]. Recently, several reports were published saying that natural vitamin $\mathrm{E}$ has a more beneficial

\footnotetext{
* Correspondence to: K. Vachiraanan (email: kanja.v@hotmail. com)
}

effect than the intake of synthetically produced vitamin $\mathrm{E}$. The main compounds of vitamin $\mathrm{E}$ in crude palm oil are $\alpha$-tocopherol and tocotrienols.

There are many separation processes for extracting tocopherols due to their complexity, their high instability when exposed heat, acids, alkalis, UV light and oxygen [5, 6]. Saponification is one of the methods which may affect the deterioration of tocopherols during heating as other studies have reported that alkali may destroy and/or change the structure of tocopherols [7-10]. Although this method is responsible for loss of tocopherols, it is the conventional extraction technique that has often been used to remove triglycerides, easily exploited and can be used in further studies $[10,11]$. The importance of this study comes from the nature of components in crude palm oil itself, in that crude palm oil is constituted of about $95 \%$ 
triglycerides. Therefore, it is difficult to separate tocopherols directly from crude palm oil. As mentioned above, focus will be on the saponification-separation technique for tocopherols from crude palm oil to solve this problem by hybrid separation process. Crude palm oil requires slight chemical modification mainly saponification and provides a better understanding of $\alpha$-tocopherol stability obtained from saponification in crude palm oil. Moreover, it was to evaluate the optimized conditions for extraction and separation of $\alpha$-tocopherol from crude palm oil. Consideration of the optimized condition was based on $\alpha$-tocopherol yield. Moreover, the optimal conditions would be used in the next step with ultrafiltration membrane process to remove triglyceride from the sample.

\subsection{METHODS/THEORY}

\subsection{Crude Palm Oil and Reagents}

The crude palm oil was kindly provided by Surattanee Palm Oil Industrial (Thailand) because it still provided good results of tocopherol yield in contrast to the crude palm oil obtained from three other mills after preliminary tocopherol yield testing was done. The samples of crude palm oil were determined by various parameters such as FFA (National Cottonseed Products-AOAC Method), saponification value (Titrimetric Method: Final action), peroxide value (American Oil Chemists' Society-AOAC Method) and $\alpha$-tocopherol content (HPLC analysis). Finally these results were compared with those summarized in the tocopherol yields.

Standard and internal standard ( $\alpha$-tocopherols and $\alpha$-tocopherols acetate respectively) were purchased from Sigma. Mobile phases such as methanol and acetronitrile used HPLC grade [12, 13]. All other reagents used analytical grade such as absolute ethanol, potassium hydroxide, sodium chloride and hexane.

\subsection{Saponification Process}

For saponification, the raw CPO from Suratthani was weighted it in a Duran tube and then ascorbic acid in absolute ethanol and potassium hydroxide solution (concentration range: $10-60 \% \mathrm{w} / \mathrm{v}$ ) were added to the sample. The tubes were incubated at $60-80^{\circ} \mathrm{C}$ for $20-40$ minutes. After saponification, the saponified was incompletely separated from unsaponified. The top layer of the solution is unsaponified. Therefore, the saponified is the lower layer which forms soap and emulsion. Then, sodium chloride was added after cooling to completely separate the saponified from the unsaponified mixture. The suspension was extracted three times with portions of hexane in the ratio of 1:6 CPO:solvent. After extraction with hexane, the nutraceutical compounds were dissolved in hexane (upper level) and the residue was separated in the lower level. The upper phase (dissolved in hexane) was separated and put into a flask, followed by the next step which was preparing to evaporate by vacuum-evaporator. The organic phase was evaporated to dryness at $40^{\circ} \mathrm{C}$ and the residue was dissolved in $1.0 \mathrm{ml}$ of chloroform. Internal standard solution ( $\alpha$ tocopherol acetate in chloroform) was added to the samples, after passing through a $0.2 \mu \mathrm{m}$ syringe filter nylon. After that, the $\alpha$-tocopherol content was measured by reversed-phase highperformance liquid chromatrography. It was kept at $-20^{\circ} \mathrm{C}$ before injection but only remained stable for a week [14].

\subsection{Membrane Filtration Unit}

Flat-sheet membrane was made from PVDF (hydrophobic membrane), of molecular weight $75 \mathrm{kDa}$ (PVDF400 sheet membrane PPC fabric, U.S.A.). The membrane presented an effective filtering area of $6.94 \times 10^{-3} \mathrm{~m}^{2}$ (diameter of $4.7 \mathrm{~cm}$ ) and rejected $95 \%$ with $2000 \mathrm{ppm}$ concentration of marker. The membrane was studied for it effectiveness in separating triglycerides from the mixture. The optimum conditions for saponification process were used for these separation studies. For ultrafiltration, the sample from the optimal saponification condition was used in this step. Since the optimal conditions could not remove all saponifiable substances such as triglycerides and phospholipids, even if it gave a high yield of tocopherols, so ultrafiltration was used after the 


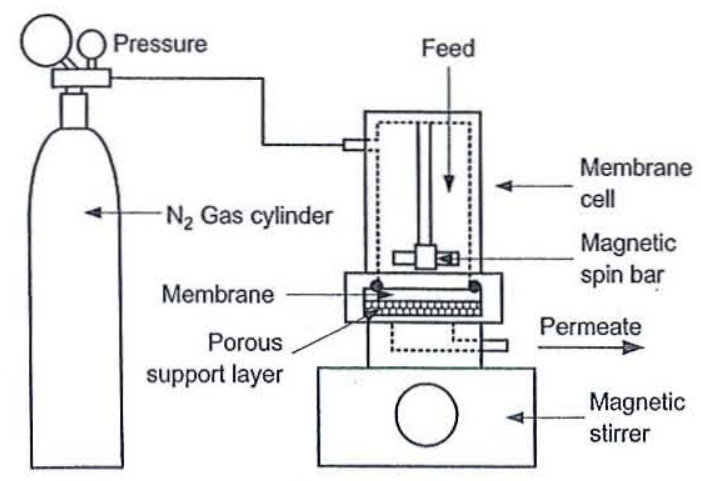

Figure 1 Membrane apparatus

reaction step of saponification instead of evaporated organic solvent (hexane) for rejecting the residue of saponified substances from the sample. Experiments were performed in a $150 \mathrm{ml}$ stirred dead-end cell pressurized by nitrogen, as shown in Figure 1. In all experiments, the temperature used was $25^{\circ} \mathrm{C}$ and pressure varied from 0.25 to 1 bar. Each experiment was performed in batch mode. Permeate samples were collected and weighed in regular time intervals in order to determine the permeate flux.

\subsection{HPLC Analysist}

HPLC separation was performed with a HewlettPackard liquid chromatographic system (Waldbron, Germany) equipped with a HP-1050 pump system, and a Rheodyne Model 7125 injector (Cotati, CA, USA) with a final loop volume of $500 \mu \mathrm{l}$. A HP-1040M UV-visible detection (VWD) system was also used. The data was stored and processed by an HPLC Chemstation (Dos Series, Hewlett-Packard). The column was a stainless steel Zorbax SB-C18 $(150 \times 4.6$ I.D., $5 \mu \mathrm{m}$ particle size).

The injection volume was $20 \mu \mathrm{L}$. The mobile phase was methanol:acetronitrile $(70: 30, \mathrm{v} / \mathrm{v})$ and the elution was performed at a flow rate of $1 \mathrm{ml} /$ $\min$. The analytical column was kept at $\mathrm{T}=25^{\circ} \mathrm{C}$. To determine the compounds in the samples and peak area ratios were used for calculations following the internal standard method. Detection was performed at $292 \mathrm{~nm}$ and each run lasted $8.6 \mathrm{~min}$.

\subsection{RESULTS AND DISCUSSIONS}

\subsection{Optimal Saponification Conditions}

Results of tocopherol yields by varying $\mathrm{KOH}$ concentrations show in Figure 2 that decreasing tocopherol yields was observed with the increase in $\mathrm{KOH}$ concentration from $10-60 \%$. It was observed that the higher concentration of alcoholic $\mathrm{KOH}$ has a remarkable effect on $\alpha$ tocopherol content. 95\% removal of triglycerides was reached when the concentration of alcoholic $\mathrm{KOH}$ was about $60 \%$. This is not surprising because the higher concentration of alcoholic $\mathrm{KOH}$ is responsible for removing triglycerides and phospholipids. However, it had significant effect on the extraction of $\alpha$-tocopherol content. The reason for this is related to alkali that may destroy and/or change the structure of tocopherols. Therefore, the great increase in deterioration of $\alpha$-tocopherols was found in the limit of high concentration alcoholic $\mathrm{KOH}$.

Based on the $\alpha$-tocopherol yields, the optimum saponification condition was at $10 \%$ alcoholic $\mathrm{KOH}$ with a minimum loss of about $18 \% \alpha$-tocopherol although this condition did not remove all triglycerides. Therefore, the coupling of another separation unit should be employed in the advanced stage to separate triglycerides and other molecules. Figure 3 compares the $\alpha$-tocopherol yields with times and temperatures at the $10 \% \mathrm{KOH}$ concentration. As can be seen, the operating parameters, time and temperature, were not significant in $\alpha$-tocopherol loss compared to the concentration of alcoholic $\mathrm{KOH}$.

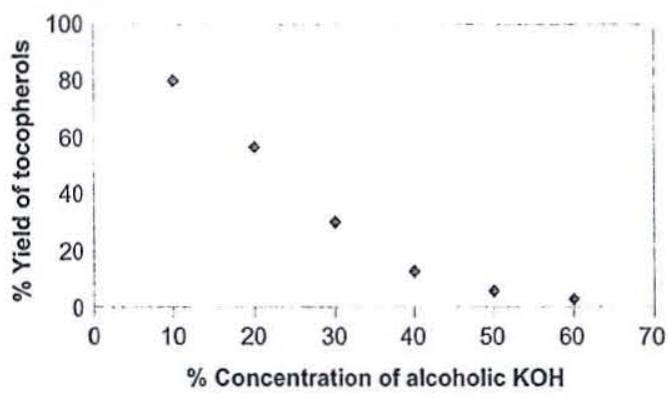

Figure 2 Yields of $\alpha$-tocopherols by saponification under different concentration of alcoholic KOH 10-60\% 


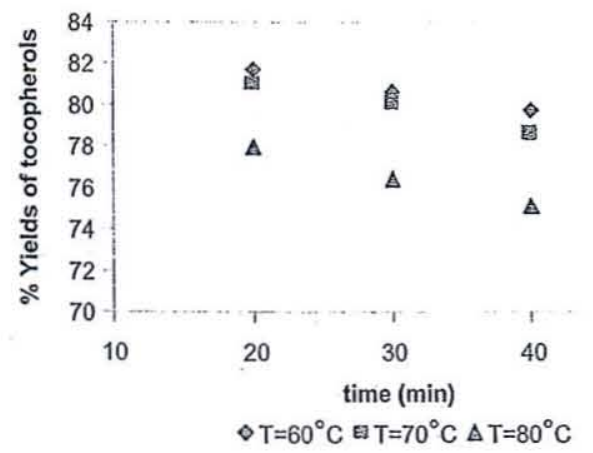

Figure 3 Yields of $\alpha$-tocopherols by saponification under various conditions $\left(\mathrm{T}=60-80^{\circ} \mathrm{C}\right.$ and time $=20-40 \mathrm{~min}$ ) .

For saponification reaction, it showed that higher temperature corresponds with loss of tocopherols. Therefore, the most suitable temperature for this study is at $\mathrm{T}=70^{\circ} \mathrm{C}$, as shown in Figure 3. The saponification time results provided the same trends as temperature results, that is, suitable time should be determined to obtain the optimal $\alpha$-tocopherol yields. The optimum saponification time in this study is at time $=30$ min, as shown in Figure 3.

As can be concluded in this part, the alcoholic $\mathrm{KOH}$ concentration plays the most significant variable compared to time and temperature. Based on the $\alpha$-tocopherol yield in this work, the optimum condition of the saponification sample at alcoholic $\mathrm{KOH} 10 \%$ is at $\mathrm{T}=70^{\circ} \mathrm{C}$ and time $=30$ $\min$. However this condition can not remove all triglycerides even it provides a high yield of $\alpha$ tocopherols. Therefore, the coupling of the membrane separation unit should be employed in the next step.

\subsection{Ultrafiltration Process}

From the optimized conditions of saponification, all triglycerides were not removed after the reaction step. So the hexane phase of saponification process was passed by ultrafiltration membrane to separate the residue of triglycerides and some minor components from this mixture. It was found that ultrafiltration can remove the large particle size residue such as triglycerides from the mixture as shown in Figure 4. The

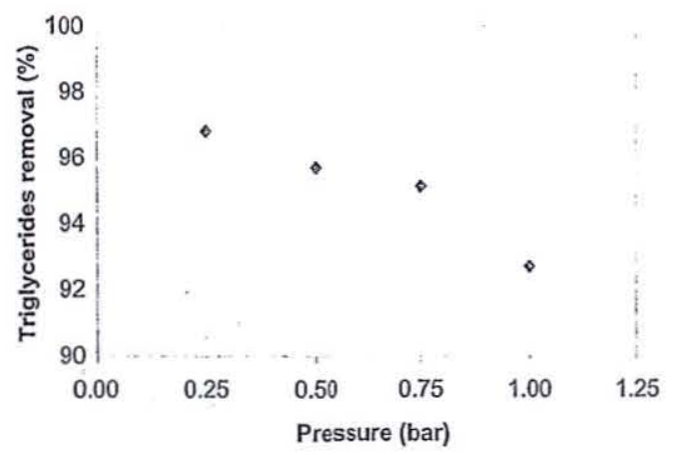

Figure 4 Effect of pressure on triglycerides removal at $\mathrm{T}=25^{\circ} \mathrm{C}$

removal efficiency of triglycerides decreased at higher pressure. In contrast, \% yield of tocopherols in the permeate was high when using the TMP at 1 bar as compared to $0.25-0.75$ bar, as shown in Figure 5. Because of that lower pressure, it led to a random arrangement of molecules. It formed a gel on the membrane surface and this phenomena played a key role as the second membrane to retain triglyceride macromolecules of and some minor components.

Figure 6 shows the effect of time processing on permeate flux. A decrease in permeate flux was observed in the initial period of filtration time. In this case in particular it is believed that during filtration, a reduction in permeate flux was due to the polarization layer which usually leads to the formation of a gel on the membrane surface and extensive fouling [1].

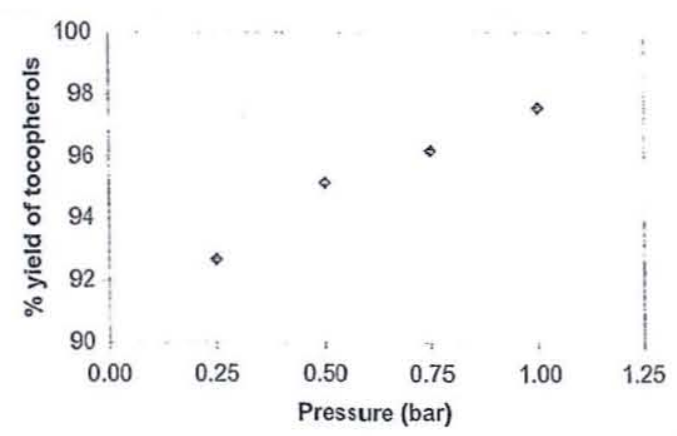

Figure 5 Effect of pressure on \% yield of tocopherols at $\mathrm{T}=25^{\circ} \mathrm{C}$ 


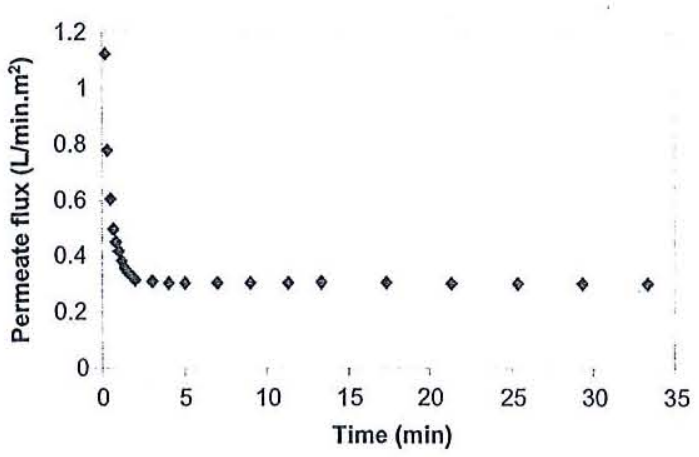

Figure 6 Effect of processing time on permeate flux at $\mathrm{T}=25^{\circ} \mathrm{C}$ and 1 bar pressure

The results showed that the optimum in saponification condition was $10 \%$ alcoholic $\mathrm{KOH}$ with a minimum loss of around $19 \% \alpha$ tocopherol. A lab scale filtration unit with hydrophobic sheet membrane was operated under frontal filtration mode at room temperature. The results pointed out that ultrafiltration could remove most residual triglycerides and phospholipids contained in unsponifiables at TMP $0.25-1.0$ bars. It can be concluded that the optimum pressure in this ultrafiltration study was in the range of 0.75 to 1 bar under the consideration of \% yield of tocopherols, \% removal of triglyceride and flux decline. The removal of triglycerides and phospholipids reached $90 \%$ and $70 \%$ when the mixture was ultrafiltered. In addition, this hybrid process was one of the best options for $\alpha$-tocopherol purification losing about $5 \%$ of yield.

\subsection{CONCLUSIONS}

After the saponification process, the oil specimen was in the form of transparent liquid without any precipitate.This implies that ultrafiltration can be applied under frontal filtration. The results showed that the optimum condition of saponification sample at alcoholic $\mathrm{KOH} 10 \%$ is at $\mathrm{T}=70^{\circ} \mathrm{C}$ and time $=30 \mathrm{~min}$. However this condition can not remove all triglycerides even if it provides a high yield of $\alpha$-tocopherols and the best condition of triglyceride removal. Within the saponification process, $65.8 \% \mathrm{KOH}$ is required so as to saponify an exact amount of fat (triglycerides). However in order to use up all triglycerides, a higher concentration of $\mathrm{KOH}$ $(>60 \%)$ is needed and \% yield of tocopherols will be improved and better when the next step of purification is investigated using nanofiltration and also reverse osmosis.

\section{REFERENCES}

[1] Juliana, M.L., N. de Moura, Lireny, and A.G. Goncalves. 2005. Degumming of Vegetable Oil by Microporous Membrane. J. Food Eng. 70: 473-478.

[2] Olcott, H.S., and O.H. Emerson. 1973. Antioxidants and the Autoxidation of Fats IX: The Antioxidant Properties of Tocopherols. J. American Oil Chemist's Society 59: 1008-1009.

[3] Bauernfeind, J. in, L.J. Machlin. 1980. Vitamin E - A Comprehensive Treatise. New York: Marcel Dekker.

[4] Burton, G.W., and Ingold, K.U. 1981. Antioxidant Activity of Vitamin E and Related Chain-breaking Phenolic Antioxidants in Vitro. J. American Chemist's Society. 103: 6472-6477.

[5] Park, S-R., Y-H Kim, H-J Park, and Y-S Lee. 2002. Stability of Tocopherols and Tocotrienols Extracted from Unsaponifiable Fraction of Rice Bran Under Various Temperature and Oxygen Condition.

[6] Sanchez-Machado, D.I., J. Lopez Cervantes. 2006. High-performance Liquid Chromatography Method for the Simultaneous Quantification of Retinol, $\alpha$-tocopherol, and Cholesterol in Shrimp Waste Hydrolysate. J. Chromatography A. 1105: 135-139.

[7] Di Mascio, P., M.E. Murphy, and H. Sies. 1991. Antioxidant Defense Systems: The Role of Carotenoids, Tocopherols and Thiols. American J. Clinical Nutrition. 53: 194-200.

[8] Gimeno, E., A.I. Castellote, R.M. LamuelaRaventos, M.C. de la Torre, and M.C. Lopez-Sabate. 2000. Rapid Determination 
of Vitamin E in Vegetable Oils by Reversed Phase High-performance Liquid Chromatography. J. Chromatography A. 881: 251-254.

[9] Ruperez, F.J., C. Barbas, S. Martinez, and E. Herrera. 1998. Rapid Determination by Reversed-phase High Performance Liquid Chromatography of Vitamins A and E in Infant Formulas. J. Chromatography A. 823: 483.

[10] Dionisi, F., J. Prodolliet, and E. Tagliaferri. 1995. Assesment of Olive Oil Adulteration by Reversed-phase High-performance Liquid Chromatography/amperometric Detection of Tocopherols and Tocotrienols. J. American Oil Chemists Society. 1505.

[11] Lietz, G., and C.J.K. Henry. 1997. A Modified Method to Minimise Losses of Carotenoids and Tocopherols during HPLC Analysis of Red Palm Oil. J. Food Chemistry. 60: 109.
[12] Khachick, F., G.R. Beecher, N.F. Whitaker. 2000. The Potential for the Improvement of Carotenoid Levels in Foods and the Likely Systemic Effects. J. Agricultural and Food Chem. 54: 101.

[13] Nierenberg, D.W., D.C. Laser. 1985. Extraction of Fat-soluble Vitamins. J. Chromatography. 345: 275.

[14] Epler, K.S., and L.C. Sander. 1992. Separation of Carotenoids by High-performance Liquid Chromatography with Polymeric and Monomeric Octadecylsilica Stationary Phases. J. Chromatography. 595: 89.

[15] Gimeno, E., E. Calero, A.I. Castellote, R.M. Lamuela-Raventos. 2000. Simultaneous Determination of $\alpha$-tocopherol and $\beta$ carotene in Olive Oil by Reversed-phase High-performance Liquid Chromatography. J. Chromatography A. 881: 255-259 